\title{
The effect of DPP-4 inhibitors on asthma control: an administrative database study to evaluate a potential pathophysiological relationship
}

This article was published in the following Dove Press journal:

Pragmatic and Observational Research

\author{
Gene Colice' \\ David Price ${ }^{2,3}$ \\ Maria Gerhardsson de \\ Verdier ${ }^{4}$ \\ Karma Rabon-Stith ${ }^{5}$ \\ Christopher Ambrose ${ }^{5}$ \\ Katherine Cappell ${ }^{6}$ \\ Debra E Irwin ${ }^{7}$ \\ Paul Juneau ${ }^{7}$ \\ Anna Vlahiotis ${ }^{7}$ \\ In collaboration with \\ Respiratory Effectiveness \\ Group (REG) \\ 'Global Medicines Development, \\ AstraZeneca, Gaithersburg, MD, USA; \\ ${ }^{2}$ Observational \& Pragmatic Research \\ Institute, Singapore; ${ }^{3}$ Centre of \\ Academic Primary Care, University \\ of Aberdeen, Aberdeen, UK; ${ }^{4} \mathrm{Global}$ \\ Medical Affairs, AstraZeneca, Mölndal, \\ Sweden; ${ }^{5} \mathrm{Global}$ Medical Affairs, \\ AstraZeneca, Gaithersburg, MD, \\ USA; ${ }^{6}$ Custom Data Analytics, Life \\ Sciences, Truven Health Analytics, an \\ IBM Watson Health Company, Ann \\ Arbor, MI, USA; ' Outcomes Research, \\ Life Sciences, Truven Health Analytics, \\ an IBM Watson Health Company, \\ Bethesda, MD, USA
}

Rationale: DPP-4 may regulate immunological pathways implicated in asthma. Assessing whether DPP-4 inhibitor (DPP-4i) use might affect asthma control is clinically important because DPP-4i use in type 2 diabetes mellitus management (T2DM) is increasing. This study evaluated associations between DPP-4i use and asthma control.

Methods: This was a retrospective, observational, matched cohort study using administrative claims in the MarketScan ${ }^{\circledR}$ Commercial Claims and Encounters (Commercial) and Medicare Supplemental and Coordination of Benefits (Medicare Supplemental) databases. Adult asthma patients initiating an oral DPP-4i or a non-DPP-4i between November 1, 2006 and March 31, 2014 were included. Patients were followed for asthma-related outcomes for 12 months after initiation of the antidiabetes medication. Outcomes included risk-domain asthma control (RDAC), defined as no asthma hospitalizations, no lower respiratory tract infections, and no oral corticosteroid (OCS) prescriptions; overall asthma control (RDAC criteria plus limited short-acting beta agonist use); treatment stability (RDAC criteria plus no increase of $\geq 50 \%$ in inhaled corticosteroid dose or addition of other asthma therapy); and severe asthma exacerbation rates (asthma-related hospitalizations, emergency room visits, or acute treatments with OCS). Comparisons were made between two matched cohorts (DPP-4i vs. non-DPP-4i initiators) using multivariable logistic regression and generalized linear modeling. Covariates included baseline demographic and clinical characteristics related to asthma and T2DM.

Results: The adjusted odds of achieving RDAC (odds ratio [OR]: 1.05; 95\% CI: 0.964 to 1.147), overall asthma control (OR: $1.04 ; 95 \%$ CI: 0.956 to 1.135$)$, and treatment stability (OR: 1.04; $95 \%$ CI: 0.949 to 1.115$)$ did not differ between the DPP-4i and non-DPP-4i cohorts. A difference was not found between cohorts in severe asthma exacerbation rates during the 12 months following initiation of antidiabetes treatment (mean $=0.32$ vs. 0.34 exacerbations per subject-year, respectively; $p=0.064$ ).

Conclusion: Asthma control was similar between patients initiating DPP-4i and non-DPP-4i antidiabetes medications, suggesting no association between DPP-4i use and asthma control.

Keywords: asthma control, DPP-4i, type 2 diabetes

\section{Introduction}

CD26 is a membrane-anchored ectoenzyme with DPP-4 activity. ${ }^{1-4}$ CD26 is found on $\mathrm{T}$ lymphocytes (more often on CD4+ than CD8+ lymphocytes), widely distributed throughout the body, in high concentrations in lung connective tissue and in lower concentrations in the apical membrane of bronchial epithelial cells and submucosal glands. DPP-4 is the circulating form of CD26 and is found in blood and biologic fluids, such as bronchoalveolar lavage. DPP-4 is the major member of the S9B subfamily of serine
Correspondence: Gene Colice AstraZeneca, 200 Orchard Ridge Drive, Gaithersburg, MD 20878, USA $\mathrm{Tel}+\mathrm{I} 3013980582$

Email Gene.Colice@astrazeneca.com 
proteases, which cleave N-terminal dipeptides from proteins with a penultimate proline or alanine. DPP-4 plays an important role in glucose homeostasis by inactivating incretins. Incretin hormones increase glucose-dependent secretion of insulin, which reduces post-prandial plasma glucose. In patients with type 2 diabetes mellitus (T2DM), therapeutic inhibition of DPP-4 reduces proteolysis and increases intact post-prandial incretin levels, thereby decreasing blood glucose..$^{5}$

DPP-4 activity may regulate immunological pathways implicated in asthma by interacting with key mediators and by inactivating chemokines. ${ }^{1}$ In vitro DPP-4 cleaves eotaxin and down-regulates its eosinophilic chemotactic potency. ${ }^{6,7}$ In a rat asthma model, DPP-4 inactivated eotaxin and reduced eosinophil chemotaxis. ${ }^{7}$ In this model, DPP-4 deficient rats had enhanced eosinophil chemotaxis. DPP-4 also inhibits chemotaxis by inactivating RANTES. ${ }^{8}$ A study in CD26 knockout mice showed enhanced airway inflammation and increased levels of eotaxin and RANTES with ovalbumin sensitization. ${ }^{9}$ Other research, though, showed that DPP-4 inhibition led to reduced airway inflammation. ${ }^{10}$ Immunohistochemistry techniques showed DPP-4 staining in airway biopsies of asthma patients. Shiobura et al found greater expression of DPP-4 in bronchial epithelial cells, ${ }^{11}$ but van der Velden et al did not confirm this finding in a similar study. ${ }^{12}$ Elevated membrane DPP-4 expression has been observed on $\mathrm{T}$ cells in asthma patients. ${ }^{13}$ A role for DPP-4 in inflammatory respiratory diseases such as asthma has thus been suggested by Giovannini-Chami et al ${ }^{14}$ who found elevated DPP-4 transcripts in the nasal epithelia of children with dust mite allergic rhinitis, which is known to be associated with uncontrolled asthma. From these varied observations it is unclear if DPP-4 activity plays a role in either up- or down-regulating key aspects in asthma pathophysiology.

The potential role of DPP-4 in asthma pathophysiology is a clinically important issue because of the increasing use of DPP-4 inhibitors (DPP-4is) to manage T2DM patients, some of whom may have concomitant asthma. To test the hypothesis that DPP-4i use affects asthma control, this study compared asthma outcomes between asthma patients newly initiating treatment with DPP-4i vs. other antidiabetic medications over a 1-year follow-up period using well-established administrative database methods. ${ }^{15-18}$ The hypothesis tested in this study was identified a priori with a commitment to publish the findings of the study.

\section{Materials and methods}

\section{Data sources}

This was a retrospective, observational, matched cohort study based on US administrative claims data extracted from the MarketScan ${ }^{\circledR}$ Commercial Claims and Encounters (Commercial) and Medicare Supplemental and Coordination of Benefits (Medicare Supplemental) databases. These databases contain enrollment information, demographic information, and inpatient medical, outpatient medical, and outpatient pharmacy claims data. The Commercial database includes information for individuals under the age of 65 years who are the primary insured or a spouse or dependent thereof. The Medicare Supplemental database includes information for individuals who are Medicare-eligible (primarily those over the age of 65 years) and have supplemental insurance paid for by their current or former employer. The Medicare Supplemental database includes both the Medicare-paid and supplemental-paid components of reimbursed administrative claims. As described in greater detail in the following section, study data were collected from the database using enrollment records, International Classification of Diseases, 9th Revision, Clinical Modification (ICD-9-CM) codes, Current Procedural Technology 4th edition codes, Healthcare Common Procedure Coding System codes, and National Drug Codes, as appropriate. All study data were accessed with protocols compliant with US patient confidentiality requirements, including the Health Insurance Portability and Accountability Act of 1996 (HIPAA) regulations. As this study used only de-identified patient records, it was exempted from Institutional Review Board approval.

\section{Inclusion/exclusion criteria}

The pre-specified study population comprised adult asthma patients with comorbid T2DM who were newly initiating either an oral DPP-4i or a non-DPP-4i antidiabetes medication. Patients were included in the study if they had at least one outpatient pharmacy claim for a DPP-4i or another non-DPP-4i antidiabetic medication not including metformin or insulin between November 1, 2006 and March 31,2014 . The date of the first of such pharmacy claims for a DPP-4i or non-DPP-4i was designated as the index date; the identity of the medication class on this claim determined the index medication class. Patients were also required to be at least 18 years old on the index date, have at least 1 year of continuous enrollment in medical and pharmacy benefits prior to the index date (designated the baseline period), and have at least 1 year of continuous enrollment in medical and pharmacy benefits after the index date (designated the follow-up period).

Included patients were also required to have evidence of an asthma diagnosis as indicated by meeting at least one of the following criteria in the baseline period or on the 
index date: 1) at least one inpatient claim or one emergency department (ED) claim with a primary diagnosis for asthma (ICD-9-CM code 493.0x, 493.1x, or 493.9x) as specified in the Healthcare Effectiveness Data and Information Set (HEDIS) claims-based definition for persistent asthma. ${ }^{19}$ Given the potential for misclassification using the HEDIS criteria, asthma was also identified in patients with; 2) two or more other (i.e., non-ED) outpatient medical claims with an asthma diagnosis or; 3 ) at least one inpatient or outpatient medical claim with an asthma diagnosis along with at least one outpatient prescription claim for an asthma medication, including immunomodulators, inhaled corticosteroids (ICS), long-acting beta agonists (LABAs), ICS/LABA combinations, leukotriene modulators, mast cell stabilizers, short-acting beta agonists (SABAs), or xanthines.

Two mutually exclusive study cohorts were defined from the study population. All patients fell into one of the two following cohorts: the DPP-4i cohort comprised patients who newly initiated alogliptin, linagliptin, saxagliptin, sitagliptin, or combination products (e.g., pioglitazone/ sitagliptin) on the index date; the non-DPP-4i cohort comprised patients who newly initiated an alpha-glucosidase inhibitor, amylin analog, glucagon-like peptide-1 receptor agonist, meglitinide, sodium-glucose co-transporter 2 inhibitor, sulfonylurea, and/or thiazolidinedione on the index date. Metformin was not included as a non-DPP-4i index medication because of the widespread prevalence of use of metformin as first-line antidiabetes therapy. ${ }^{20}$ Followup after the initial exposure (index date) was conducted in an intention-to-treat manner.

Patients in either cohort were excluded from the study if they had an outpatient prescription drug claim for a DPP-4i during the baseline period. Patients in the non-DPP-4i cohort were excluded if they had any outpatient prescription drug claims for any medication in the same non-DPP-4i medication class as their index prescription during the baseline period. Patients in both groups were excluded if they had a medical claim with an ICD-9-CM diagnosis code indicative of COPD or chronic respiratory tract disease other than asthma during the baseline or follow-up periods.

\section{Study variables}

The primary independent variable was a dichotomous indicator for membership in the DPP-4i or non-DPP-4i cohort. Baseline measures of patient demographics, general health status, asthma and diabetes severity, comorbid conditions, along with asthma and diabetes medication utilization were also collected.
Study outcomes were risk-domain asthma control (RDAC), overall asthma control, treatment stability, and number of severe asthma exacerbations, which were rospectively defined as follows: ${ }^{18}$

- $\mathrm{RDAC}$ during the 12-month period following the index date (yes/no): a composite measure based on a patient having no recorded asthma-related hospital attendance, including inpatient admission, ED attendance, or outpatient hospital attendance; no outpatient consultation for lower respiratory tract infection (LRTI) with a resultant antibiotic prescription; and no outpatient prescriptions for an acute course of oral corticosteroids (OCS) during the follow-up period. Asthma-related hospitalization was considered as any admission or ED attendance with an ICD-9-CM diagnosis indicative of either asthma or LRTI in any diagnosis position. An acute course of OCS was specifically defined as a prescription with days supplied less than or equal to 14 days or with days supplied greater than 14 days and daily dosage greater than $10 \mathrm{mg}$. LRTI requiring antibiotics were infections for which an antibiotic was prescribed on the same day as the recorded diagnosis.

- Overall asthma control during the 12-month period following the index date (yes/no): a composite measure comprising the three previously mentioned criteria for RDAC, plus limited SABA use (average daily dose of $\leq 180 \mu \mathrm{g}$ albuterol equivalents) as a fourth criterion.

- Treatment stability during the 12-month period following the index date (yes/no): a composite measure comprising the three previously mentioned criteria for RDAC, plus no asthma treatment change as a fourth criterion. Asthma treatment change was specifically defined as either increased ICS dose (average daily dose increased by greater than or equal to $50 \%$ from the baseline period to the followup period) or use of additional asthma therapy, such as a LABA, leukotriene receptor antagonist, or theophylline.

- Severe asthma exacerbations during the 12-month period following the index date were defined as either an asthmarelated hospital admission, ED attendance, or an acute outpatient course of OCS. Any such events within a 2-week window were counted as a single exacerbation. For asthmarelated hospitalizations and ED attendance, diagnoses of asthma were permitted to appear in any diagnosis position.

\section{Statistical analysis}

Patients in the DPP-4i cohort were directly matched to those in the non-DPP-4i cohort on demographic and baseline covariates that were hypothesized to possibly affect study 
outcomes, with the exception of patient age, which was matched using an age range. Patients were considered a suitable match if their respective ages were within 5 years of one another. Other factors matched directly were sex, baseline number of acute OCS prescriptions, baseline SABA daily dose, baseline ICS daily dose, and baseline adapted Diabetes Complication Severity Index (aDCSI) ${ }^{21}$ score. DPP-4i patients were matched to non-DPP-4i patients at a 1:2 ratio, with 1:1 matched pairs substituted where 1:2 matching was not possible. Patients were matched before outcomes were examined. The analysis was conducted on an intention-to-treat basis in that patients who were classified into the non-DPP-4i cohort based on new initiation of a non-DPP-4i medication at index, but who subsequently filled a prescription for a DPP-4i after the index date, were not excluded.

RDAC, overall asthma control, and treatment stability during the follow-up period were compared between the matched DPP-4i and non-DPP-4i cohorts, treating the nonDPP-4i cohort as the reference category, with individual standard logistic regression models with a unique outcome as a dependent variable, controlling for other covariates. Odds ratios (ORs) from logistic regression models, along with their $95 \%$ CIs, were computed for the pre-specified binary outcomes. The rate of severe asthma exacerbations during the follow-up period was compared between the matched DPP-4i and non-DPP-4i cohorts, treating the non-DPP-4i cohort as the reference category, through a generalized linear regression model, assuming an underlying negative binomial distribution. Covariates included in the logistic and negative binomial regression models were demographic characteristics not used for direct matching, diabetes- and asthma-related comorbidities, all diabetes- and asthma-related medication history measures not used for direct matching, and the DeyoCharlson Comorbidity Index (DCI). ${ }^{22}$ No offset was used for the negative binomial model, as all patients were followed for a fixed 1-year follow-up period. For all outcomes, a $p$-value of $p<0.05$ was used to denote statistical significance.

\section{Results}

A total of 4,304 patients met all of the criteria for inclusion in the DPP-4i cohort, and 12,659 patients met criteria for inclusion in the non-DPP-4i cohort. There were 916 (7.2\%) non-DPP-4i patients who filled at least one prescription for a DPP-4i after the index date. After matching, 3,973 patients remained in the DPP-4i cohort and 7,487 patients remained in the non-DPP-4i cohort $(n=3,5141: 2$ match ratio; $n=459$ 1:1 match ratio). Baseline demographic characteristics were similar between the groups (Table 1). Mean age was 53.7 years for the DPP-4i cohort and 53.4 years for the non-DPP-4i cohort. Approximately $70 \%$ of the patients were females in each of the matched cohorts. Although there were significant differences between the two cohorts with regards to some baseline patient characteristics (health insurance plan type, geographic region, and index year), these differences were considered small and not clinically meaningful.

The DPP-4i cohort had significantly higher mean DCI scores $(2.22$ vs. $2.11, p<0.05)$ and mean aDCSI scores $(0.53$ vs. $0.49, p<0.05)$ at baseline compared with the non-DPP-4i

Table I Patient characteristics

\begin{tabular}{|c|c|c|c|}
\hline Characteristic & $\begin{array}{l}\text { DPP-4i } \\
N=3,973\end{array}$ & $\begin{array}{l}\text { Non-DPP-4i } \\
N=7,487\end{array}$ & $p$-value \\
\hline Age (Mean, SD)' & $53.7(10.5)$ & $53.4(10.3)$ & 0.150 \\
\hline $\operatorname{Sex}(\mathbf{N}, \%)^{\prime}$ & & & 0.563 \\
\hline Male & $\mathrm{I}, 244$ (3I.3\%) & $2,305(30.8 \%)$ & \\
\hline Female & 2,729 (68.7\%) & $5,182(69.2 \%)$ & \\
\hline $\begin{array}{l}\text { Health insurance plan } \\
\text { type }(\mathbf{N}, \%)\end{array}$ & & & $<0.001$ \\
\hline Comprehensive & $304(7.7 \%)$ & $55 \mathrm{I}(7.4 \%)$ & \\
\hline EPO & $50(1.3 \%)$ & $81(1.1 \%)$ & \\
\hline $\mathrm{HMO}$ & $514(12.9 \%)$ & I,374 (I8.4\%) & \\
\hline POS & $320(8.1 \%)$ & $57 \mid(7.6 \%)$ & \\
\hline PPO & $2,410(60.7 \%)$ & $4,308(57.5 \%)$ & \\
\hline POS with capitation & $25(0.6 \%)$ & $32(0.4 \%)$ & \\
\hline $\mathrm{CDHP}$ & $161(4.1 \%)$ & $210(2.8 \%)$ & \\
\hline $\mathrm{HDHP}$ & 48 (I.2\%) & $76(1.0 \%)$ & \\
\hline Other/unknown & $|4|$ (3.5\%) & $284(3.8 \%)$ & \\
\hline Payer (N, \%) & & & 0.414 \\
\hline Commercial & 3,487 (87.8\%) & $6,610(88.3 \%)$ & \\
\hline Medicare & $486(12.2 \%)$ & $877(11.7 \%)$ & \\
\hline Geographic region ( $N$, \%) & & & $<0.001$ \\
\hline Northeast & 871 (21.9\%) & I, I 72 (I5.7\%) & \\
\hline North Central & $857(21.6 \%)$ & I,786 (23.9\%) & \\
\hline South & $\mathrm{I}, 604(40.4 \%)$ & $2,950(39.4 \%)$ & \\
\hline West & 604 (I5.2\%) & I,509 (20.2\%) & \\
\hline Unknown & 37 (0.9\%) & 70 (0.9\%) & \\
\hline Population density (N, \%) & & & 0.502 \\
\hline Urban & $3,384(85.2 \%)$ & $6,320(84.4 \%)$ & \\
\hline Rural & $554(13.9 \%)$ & I,I04 (14.7\%) & \\
\hline Unknown & $35(0.9 \%)$ & $63(0.8 \%)$ & \\
\hline Index year (N, \%) & & & $<0.001$ \\
\hline 2006 & $38(1.0 \%)$ & 215 (2.9\%) & \\
\hline 2007 & 374 (9.4\%) & I,067 (14.3\%) & \\
\hline 2008 & $410(10.3 \%)$ & 916 (12.2\%) & \\
\hline 2009 & 405 (10.2\%) & I,05I (I4.0\%) & \\
\hline 2010 & $562(14.1 \%)$ & 1,203 (16.1\%) & \\
\hline 2011 & 745 (I8.8\%) & I,II9 (14.9\%) & \\
\hline 2012 & $686(17.3 \%)$ & $926(12.4 \%)$ & \\
\hline 2013 & $623(15.7 \%)$ & $792(10.6 \%)$ & \\
\hline 2014 & $130(3.3 \%)$ & $198(2.6 \%)$ & \\
\hline
\end{tabular}

Notes: 'Used in direct matching. The bold value denotes statistical significance $(p<0.05)$.

Abbreviations: CDHP, consumer-driven health plan; DPP-4i, DPP-4 inhibitor; EPO, exclusive provider organization; HDHP, high deductible health plan; HMO, health maintenance organization; POS, point-of-service; PPO, preferred provider organization. 
cohort (Table 2). A significantly higher proportion of patients $(p<0.05)$ in the DPP-4i cohort had rhinitis and eczema than in the non-DPP-4i cohort. Patients in the DPP-4i cohort also had significantly higher observed rates of hypertension, dyslipidemia, atherosclerosis, and peripheral vascular disease compared to the non-DPP-4i cohort (all $p<0.05$ ).

Asthma medication use during the baseline period was similar for the two cohorts (Table 3 ). Approximately $64 \%$ of patients received no OCS treatment, $23 \%$ received a single course, and $6 \%$ received three or more courses. During the baseline period, approximately $45 \%$ of patients in each cohort received no treatment with SABA and approximately 55\% in each cohort received no treatment with ICS. There was a significant difference between the two cohorts in utilization of controller medications, but the difference was small and not clinically relevant. A significantly greater proportion of DPP-4 $\mathrm{i}$ than non-DPP-4i initiators received metformin during the baseline period ( $58.8 \%$ vs. $52.8 \%, p<0.05)$, but there was no statistically significant difference between cohorts for the proportion of patients with prior exposure to insulins.

In the baseline period, there were no significant differences between the two cohorts in RDAC, overall asthma control, and number of severe asthma exacerbations (Table 4). There were significant differences in two components of the RDAC relating to inpatient admissions, ED visits, and outpatient hospital visits for asthma and LRTI, but these differences were small.

During the 1-year follow-up period, all aspects of asthma control improved compared to baseline in both cohorts, but RDAC, overall asthma control, and number of severe asthma exacerbations were similar for the DPP-4i and non-DPP-4i cohorts (Table 5). Again seen were significant differences between the two cohorts in components of the RDAC. Postperiod treatment stability (which was not measured during the baseline period) was also comparable between the DPP-4i and non-DPP-4i cohorts. Multivariable logistic regression analysis

Table 2 Baseline health status and comorbid conditions

\begin{tabular}{|c|c|c|c|}
\hline Clinical characteristic & $\begin{array}{l}\text { DPP-4i } \\
N=3,973\end{array}$ & $\begin{array}{l}\text { Non-DPP-4i } \\
N=7,487\end{array}$ & $p$-value \\
\hline \multicolumn{4}{|l|}{ General health status } \\
\hline Deyo-Charlson Comorbidity Index (Mean, SD) & $2.22(1.2)$ & $2.11(1.1)$ & $<0.0001$ \\
\hline Adapted Diabetes Complication Score Index (Mean, SD)' & $0.53(1.0)$ & $0.49(0.9)$ & 0.015 \\
\hline \multicolumn{4}{|l|}{ Comorbid conditions } \\
\hline Hypertension & $2,700(68.0 \%)$ & $4,927(65.8 \%)$ & 0.020 \\
\hline Dyslipidemia & $2,443(61.5 \%)$ & $4,215(56.3 \%)$ & $<0.0001$ \\
\hline Rhinitis & $1,091(27.5 \%)$ & I,80I (24.1\%) & $<0.000$ I \\
\hline Gastroesophageal reflux disease & $540(13.6 \%)$ & $925(12.4 \%)$ & 0.059 \\
\hline Atherosclerosis & $380(9.6 \%)$ & $633(8.5 \%)$ & 0.046 \\
\hline Ischemic heart disease & $349(8.8 \%)$ & $633(8.5 \%)$ & 0.549 \\
\hline Eczema & $348(8.8 \%)$ & $567(7.6 \%)$ & 0.026 \\
\hline Depression & $344(8.7 \%)$ & $729(9.7 \%)$ & 0.059 \\
\hline Anxiety & $284(7.1 \%)$ & $522(7.0 \%)$ & 0.726 \\
\hline Diabetic peripheral neuropathy & $178(4.5 \%)$ & $323(4.3 \%)$ & 0.679 \\
\hline Diabetic retinopathy & $168(4.2 \%)$ & $320(4.3 \%)$ & 0.909 \\
\hline Smoking ${ }^{2}$ & $105(2.6 \%)$ & $223(3.0 \%)$ & 0.305 \\
\hline Heart failure & $98(2.5 \%)$ & I7| (2.3\%) & 0.539 \\
\hline Peripheral vascular disease & $87(2.2 \%)$ & $121(1.6 \%)$ & 0.029 \\
\hline Renal impairment & $75(1.9 \%)$ & $143(1.9 \%)$ & 0.934 \\
\hline Obesity & $54(1.4 \%)$ & $96(1.3 \%)$ & 0.730 \\
\hline Diabetic nephropathy & $52(1.3 \%)$ & $130(1.7 \%)$ & 0.082 \\
\hline Stroke & 48 (I.2\%) & $63(0.8 \%)$ & 0.057 \\
\hline Psoriasis & $46(1.2 \%)$ & $87(1.2 \%)$ & 0.984 \\
\hline Unstable angina pectoris & $35(0.9 \%)$ & $81(1.1 \%)$ & 0.307 \\
\hline Pulmonary hypertension & $25(0.6 \%)$ & $41(0.5 \%)$ & 0.583 \\
\hline Percutaneous coronary intervention & $18(0.5 \%)$ & $39(0.5 \%)$ & 0.623 \\
\hline Anaphylaxis & $16(0.4 \%)$ & $39(0.5 \%)$ & 0.384 \\
\hline Myocardial infarction & $15(0.4 \%)$ & $43(0.6 \%)$ & 0.158 \\
\hline Coronary artery bypass graft & $6(0.2 \%)$ & $13(0.2 \%)$ & 0.777 \\
\hline
\end{tabular}

Notes: 'Used in direct matching; ${ }^{2}$ claims-based history of tobacco use/tobacco dependence. The bold value denotes statistical significance $(p<0.05)$. Abbreviation: DPP-4i, DPP-4 inhibitor. 
Table 3 Baseline medication utilization

\begin{tabular}{|c|c|c|c|}
\hline Medication utilization & $\begin{array}{l}\text { DPP-4i } \\
N=3,973\end{array}$ & $\begin{array}{l}\text { Non-DPP-4i } \\
N=7,487\end{array}$ & $p$-value \\
\hline \multicolumn{4}{|l|}{ Asthma-related medications } \\
\hline Number of acute oral corticosteroid courses' ( $\mathrm{N}, \%)$ & & & 0.324 \\
\hline 0 & $2,507(63.1 \%)$ & $4,834(64.6 \%)$ & \\
\hline 1 & $942(23.7 \%)$ & I,737 (23.2\%) & \\
\hline 2 & $287(7.2 \%)$ & $518(6.9 \%)$ & \\
\hline$\geq 3$ & $237(6.0 \%)$ & $398(5.3 \%)$ & \\
\hline Mean SABA daily dose, albuterol & & & 0.833 \\
\hline \multicolumn{4}{|l|}{ equivalents' ( $\mathrm{N}, \%)$} \\
\hline $0 \mu g$ & I,75I (44.I\%) & $3,340(44.6 \%)$ & \\
\hline $\mathrm{I}-\mathrm{I} 00 \mu \mathrm{g}$ & I,566 (39.4\%) & 2,981 (39.8\%) & \\
\hline $10 \mathrm{I}-200 \mu \mathrm{g}$ & $284(7.1 \%)$ & $502(6.7 \%)$ & \\
\hline $20 \mathrm{I}-300 \mu \mathrm{g}$ & $172(4.3 \%)$ & $318(4.2 \%)$ & \\
\hline $30 \mathrm{I}-400 \mu \mathrm{g}$ & $46(1.2 \%)$ & $75(1.0 \%)$ & \\
\hline$>400 \mu \mathrm{g}$ & $154(3.9 \%)$ & $27 I(3.6 \%)$ & \\
\hline Mean ICS daily dose, fluticasone propionate equivalents' ( $\mathrm{N}, \%)$ & & & 0.166 \\
\hline $0 \mu \mathrm{g}$ & $2,20 \mathrm{I}(55.4 \%)$ & $4,210(56.2 \%)$ & \\
\hline $1-199 \mu g$ & $1,562(39.3 \%)$ & $2,95 \mathrm{I}(39.4 \%)$ & \\
\hline $200-399 \mu g$ & $169(4.3 \%)$ & $276(3.7 \%)$ & \\
\hline $400-599 \mu g$ & $38(1.0 \%)$ & $46(0.6 \%)$ & \\
\hline $600-799 \mu g$ & I (0.0\%) & I (0.0\%) & \\
\hline$\geq 800 \mu \mathrm{g}$ & $2(0.1 \%)$ & $3(0.0 \%)$ & \\
\hline Number of asthma prescriptions, control medications ( $\mathrm{N}, \%)$ & & & $<0.0001$ \\
\hline 0 & I,639 (4I.3\%) & $3,238(43.2 \%)$ & \\
\hline I & $630(15.9 \%)$ & $1,310(17.5 \%)$ & \\
\hline 2 & $317(8.0 \%)$ & $713(9.5 \%)$ & \\
\hline 3 & $299(7.5 \%)$ & $470(6.3 \%)$ & \\
\hline 4 & $274(6.9 \%)$ & $410(5.5 \%)$ & \\
\hline$\geq 5$ & $814(20.5 \%)$ & I,346 (I8.0\%) & \\
\hline Number of asthma prescriptions, rescue & & & 0.130 \\
\hline \multicolumn{4}{|l|}{ medications (N, \%) } \\
\hline 0 & $\mathrm{I}, 685(42.4 \%)$ & $3,163(42.2 \%)$ & \\
\hline 1 & I,24I (3I.2\%) & $2,447(32.7 \%)$ & \\
\hline 2 & $502(12.6 \%)$ & $954(12.7 \%)$ & \\
\hline$\geq 3$ & $545(13.7 \%)$ & $923(12.3 \%)$ & \\
\hline Number of antibiotic courses for LRTI (N, \%) & & & 0.702 \\
\hline 0 & $3,727(93.8 \%)$ & 7,061 (94.3\%) & \\
\hline 1 & $224(5.6 \%)$ & $387(5.2 \%)$ & \\
\hline 2 & $17(0.4 \%)$ & $28(0.4 \%)$ & \\
\hline$\geq 3$ & $5(0.1 \%)$ & II (0.1\%) & \\
\hline Acetaminophen $(\mathrm{N}, \%)$ & $\mathrm{I}, 454(36.6 \%)$ & $2,882(38.5 \%)$ & 0.046 \\
\hline Non-steroidal anti-inflammatory drugs ( $\mathrm{N}, \%)$ & I, 168 (29.4\%) & $2,162(28.9 \%)$ & 0.558 \\
\hline Beta-blockers (N, \%) & $822(20.7 \%)$ & $\mathrm{I}, 609(2 \mathrm{I} .5 \%)$ & 0.318 \\
\hline \multicolumn{4}{|l|}{ Diabetes medications (N, \%) } \\
\hline Alpha-glucosidase inhibitors & $0(0 \%)$ & $2(0 \%)$ & 0.547 \\
\hline Amylin analogs & $\mathrm{I}(0 \%)$ & $\mathrm{I}(0 \%)$ & 1.000 \\
\hline Biguanides (metformin) & $2,337(58.8 \%)$ & $3,952(52.8 \%)$ & $<0.0001$ \\
\hline Glucagon-like peptide-I receptor agonists & $12(0.3 \%)$ & $8(0.1 \%)$ & 0.017 \\
\hline Insulins ${ }^{2}$ & $312(7.9 \%)$ & $647(8.6 \%)$ & 0.147 \\
\hline Short-acting & 154 (3.9\%) & $348(4.6 \%)$ & 0.055 \\
\hline Long-acting & $254(6.4 \%)$ & $502(6.7 \%)$ & 0.522 \\
\hline Combination & $53(1.3 \%)$ & $120(1.6 \%)$ & $0.26 \mathrm{I}$ \\
\hline Meglitinides and combinations & $3(0.1 \%)$ & $4(0.1 \%)$ & 0.700 \\
\hline \multicolumn{4}{|l|}{ Sodium-glucose co-transporter 2 inhibitors } \\
\hline and combinations & $0(0 \%)$ & $0(0 \%)$ & $\mathrm{n} / \mathrm{a}$ \\
\hline Sulfonylureas and combinations & $25(0.6 \%)$ & $63(0.8 \%)$ & 0.216 \\
\hline Thiazolidinediones and combinations & $36(0.9 \%)$ & $57(0.8 \%)$ & $0.4 I I$ \\
\hline
\end{tabular}

Notes: 'Used in direct matching; ${ }^{2}$ percent of patients using different insulin types is not mutually exclusive. The bold value denotes statistical significance ( $\left.p<0.05\right)$. Abbreviations: DPP-4i, DPP-4 inhibitor; ICS, inhaled corticosteroids; LRTI, lower respiratory tract infection; n/a, not applicable; SABA, short-acting beta agonist. 
Table 4 Baseline asthma control

\begin{tabular}{|c|c|c|c|}
\hline Clinical characteristic & $\begin{array}{l}\text { DPP-4i } \\
N=3,973\end{array}$ & $\begin{array}{l}\text { Non-DPP-4i } \\
N=7,487\end{array}$ & $p$-value \\
\hline \multicolumn{4}{|l|}{ Risk-domain asthma control (N, \%) } \\
\hline $\begin{array}{l}\text { Patients with no inpatient admission, emergency department, or outpatient hospital visit } \\
\text { for asthma }\end{array}$ & $3,133(78.9 \%)$ & $5,728(76.5 \%)$ & 0.004 \\
\hline $\begin{array}{l}\text { Patients with no inpatient admission, emergency department, or outpatient hospital visit } \\
\text { for LRTI }\end{array}$ & $3,816(96.0 \%)$ & $7,074(94.5 \%)$ & $<0.001$ \\
\hline Patients with no outpatient consultation for LRTI with a resultant antibiotic prescription & $3,748(94.3 \%)$ & $7,119(95.1 \%)$ & 0.085 \\
\hline Patients with no prescriptions for an acute course of oral corticosteroids & $2,507(63.1 \%)$ & $4,834(64.6 \%)$ & 0.120 \\
\hline Patients meeting all of the above criteria indicating risk-domain asthma control & $1,943(48.9 \%)$ & $3,655(48.8 \%)$ & 0.929 \\
\hline \multicolumn{4}{|l|}{ Overall asthma control ( $N, \%)$} \\
\hline $\begin{array}{l}\text { Patients with risk-domain asthma control and limited SABA use (daily dose } \leq 180 \mu \mathrm{g} \\
\text { albuterol equivalents) }\end{array}$ & I,78I (44.8\%) & $3,353(44.8 \%)$ & 0.965 \\
\hline Number of severe asthma exacerbations $(\mathbf{N}, \%)$ & & & 0.146 \\
\hline 0 & $2,264(57.0 \%)$ & $4,303(57.5 \%)$ & \\
\hline I & $\mathrm{I}, \mathrm{I} 62(29.2 \%)$ & $2,198(29.4 \%)$ & \\
\hline 2 & $320(8.1 \%)$ & $631(8.4 \%)$ & \\
\hline$\geq 3$ & $227(5.7 \%)$ & $355(4.7 \%)$ & \\
\hline Number of asthma consultations without acute oral corticosteroids $(\mathbf{N}, \%)^{\prime}$ & & & 0.007 \\
\hline 0 & $\mathrm{I}, 093(27.5 \%)$ & $2,174(29.0 \%)$ & \\
\hline I & I,727 (43.5\%) & $3,345(44.7 \%)$ & \\
\hline 2 & $662(16.7 \%)$ & $\mathrm{I}, \mathrm{I} 82(\mathrm{I} 5.8 \%)$ & \\
\hline$\geq 3$ & $491(12.4 \%)$ & $786(10.5 \%)$ & \\
\hline
\end{tabular}

Notes: 'Used in direct matching. The bold value denotes statistical significance $(p<0.05)$.

Abbreviations: DPP-4i, DPP-4 inhibitor; LRTI, lower respiratory tract infection; SABA, short-acting beta agonist.

Table 5 Follow-up asthma control

\begin{tabular}{|c|c|c|c|}
\hline Clinical characteristic & $\begin{array}{l}\text { DPP-4i } \\
N=3,973\end{array}$ & $\begin{array}{l}\text { Non-DPP-4i } \\
N=7,487\end{array}$ & p-value \\
\hline \multicolumn{4}{|l|}{ Risk-domain asthma control (N, \%) } \\
\hline $\begin{array}{l}\text { Patients with no inpatient admission, emergency department, or outpatient hospital } \\
\text { visit for asthma }\end{array}$ & $3,629(91.3 \%)$ & $6,717(89.7 \%)$ & 0.005 \\
\hline $\begin{array}{l}\text { Patients with no inpatient admission, emergency department, or outpatient hospital } \\
\text { visit for LRTI }\end{array}$ & $3,884(97.8 \%)$ & $7,266(97.0 \%)$ & 0.025 \\
\hline $\begin{array}{l}\text { Patients with no outpatient consultation for LRTI with a resultant antibiotic } \\
\text { prescription }\end{array}$ & $3,793(95.5 \%)$ & $7,173(95.8 \%)$ & 0.398 \\
\hline Patients with no prescriptions for an acute course of oral corticosteroids & $2,917(73.4 \%)$ & $5,538(74.0 \%)$ & 0.526 \\
\hline Patients meeting all of the above criteria indicating risk-domain asthma control & $2,607(65.6 \%)$ & $4,894(65.4 \%)$ & 0.788 \\
\hline \multicolumn{4}{|l|}{ Overall asthma control ( $\mathbf{N}, \%)$} \\
\hline $\begin{array}{l}\text { Patients with risk-domain asthma control and limited SABA use (daily dose } \leq 180 \mu g \\
\text { albuterol equivalents) }\end{array}$ & $2,404(60.5 \%)$ & $4,533(60.5 \%)$ & 0.970 \\
\hline \multicolumn{4}{|l|}{ Treatment stability } \\
\hline $\begin{array}{l}\text { Patients with risk-domain asthma control and no asthma medication treatment } \\
\text { change }\end{array}$ & $2,108(49.0 \%)$ & $6.098(48.2 \%)$ & 0.360 \\
\hline Number of severe asthma exacerbations $(\mathbf{N}, \%)$ & & & 0.951 \\
\hline 0 & $2,823$ (7I. $1 \%)$ & $5,3 \mid 4(7 \mid .0 \%)$ & \\
\hline I & $757(19.1 \%)$ & $1,420(19.0 \%)$ & \\
\hline 2 & $235(5.9 \%)$ & $462(6.2 \%)$ & \\
\hline$\geq 3$ & $158(4.0 \%)$ & 291 (3.9\%) & \\
\hline
\end{tabular}

Note: The bold value denotes statistical significance $(p<0.05)$.

Abbreviations: DPP-4i, DPP-4 inhibitor; LRTI, lower respiratory tract infection; SABA, short-acting beta agonist.

revealed no significant difference between the DPP-4i cohort and non-DPP-4i cohort in odds of achieving $\mathrm{RDAC}(\mathrm{OR}=1.05$, $95 \% \mathrm{CI}=0.96-1.15)$, overall asthma control (OR $=1.04,95 \%$ $\mathrm{CI}=0.96-1.14)$, and treatment stability $(\mathrm{OR}=1.03,95 \% \mathrm{CI}$
$=0.95-1.12$ ) (Figure 1). The adjusted mean rate of severe asthma exacerbations was not significantly higher in the DPP-4i cohort than in the non-DPP-4i cohort $(0.32$ vs. 0.34 exacerbations per subject-year; $p=0.064$ ) (Figure 2). 


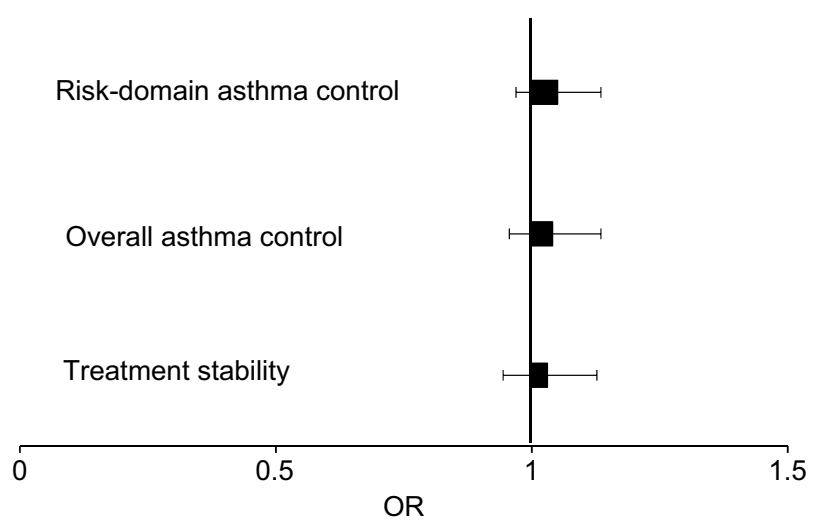

Figure I Adjusted odds of risk-domain asthma control, overall asthma control, and treatment stability during follow-up.

Note: Models adjusted for health plan type, payer, geographic region, population density, index year, anaphylaxis, anxiety, depression, eczema, gastroesophageal reflux disease, ischemic heart disease, obesity, psoriasis, pulmonary hypertension, rhinitis, smoking, dyslipidemia, hypertension, renal impairment, baseline type 2 diabetes, number of asthma control medication prescriptions, number of asthma rescue medication prescriptions, number of antibiotic courses to treat LRTI, acetaminophen utilization, NSAID utilization, beta blocker utilization, microvascular complications of diabetes, macrovascular complications of diabetes, baseline biguanide utilization, baseline insulin utilization, baseline utilization of other antihyperglycemic medications, baseline $\mathrm{DCl}$, baseline risk-domain asthma control, baseline overall asthma control, baseline number of severe asthma exacerbations and number of asthma consultations with acute OCS prescriptions.

Abbreviations: DCI, Deyo-Charlson Comorbidity Index; LRTI, lower respiratory tract infection; NSAID, non-steroidal anti-inflammatory drugs; OCS, oral corticosteroid; OR, odds ratio.

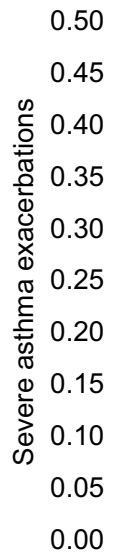

$p=0.064$

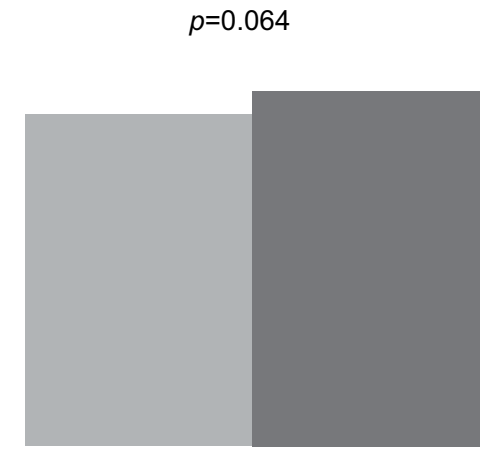

DPP-4i Cohort $\quad$ Non-DPP-4i Cohort

Figure 2 Adjusted mean estimate rate of severe asthma exacerbations during follow-up.

Note: Models adjusted for health plan type, payer, geographic region, population density, index year, anaphylaxis, anxiety, depression, eczema, gastroesophageal reflux disease, ischemic heart disease, obesity, psoriasis, pulmonary hypertension, rhinitis, smoking, dyslipidemia, hypertension, renal impairment, baseline type 2 diabetes, number of asthma control medication prescriptions, number of asthma rescue medication prescriptions, number of antibiotic courses to treat LRTI, acetaminophen utilization, NSAID utilization, beta blocker utilization, microvascular complications of diabetes, macrovascular complications of diabetes, baseline biguanide utilization, baseline insulin utilization, baseline utilization of other antihyperglycemic medications, baseline $\mathrm{DCl}$, baseline risk-domain asthma control, baseline overall asthma control, baseline number of severe asthma exacerbations and number of asthma consultations with acute OCS prescriptions.

Abbreviations: DCI, Deyo-Charlson Comorbidity Index; DPP-4i, DPP-4 inhibitor; LRTI, lower respiratory tract infection; NSAID, non-steroidal anti-inflammatory drugs; OCS, oral corticosteroid.

\section{Discussion}

Using well-established observational database research methods, this study measured, by proxy, the potential role of DDP-4 inhibition in the regulation of asthma immunology. Across several measures of asthma control, asthma outcomes for patients newly initiating a DPP-4i for the treatment of T2DM were similar to patients newly initiating non-DPP-4i antidiabetes medications suggesting no association between DPP-4i use and asthma control.

In vitro and animal studies have suggested a possible role for DPP-4 in mediating key aspects of allergic airway inflammation. ${ }^{1}$ However, we are not aware of clinical trials which have addressed the clinically relevant hypothesis that treatment with DPP-4i might affect asthma control. This analysis captured the experience of patients with both asthma and T2DM outside the limits of a clinical trial environment by utilizing real world data. Using established methods ${ }^{15-18}$ we identified a large number of asthma patients with a wide range of asthma severity, from patients with relatively mild disease not requiring regular controller medications to patients with more severe asthma and frequent exacerbations, who were also being treated for T2DM. Asthma patients have more comorbidities, like T2DM, than do non-asthma patients. ${ }^{23}$ However, clinical trials in asthma, particularly those designed to test new medications, may exclude significant proportions of the patient population potentially eligible for treatment with comorbidities like T2DM. ${ }^{24}$

Access to administrative databases allowed insights into multiple aspects of asthma care, including prescription drug usage, comorbid conditions, and health care utilization. The endpoints used in this analysis to assess asthma control, the RDAC, overall asthma control, treatment stability, and number of severe asthma exacerbations, have been well standardized in previous work ${ }^{15-18}$ and are clinically relevant. Although administrative databases do not provide access to information about asthma symptoms, SABA use has been used as a surrogate measure of this component of asthma outcomes. An interesting and unexplained observation in this study is that measures of asthma control improved in both cohorts from the baseline period to the 1-year observational period. A previous observational database study has suggested that diabetes is a predictor of future asthma exacerbations. ${ }^{25}$ Whether improved T2DM control might also improve asthma control has not been evaluated in this analysis, and warrants future study.

Other inherent strengths and weaknesses of administrative database analyses should be considered. The study databases used in this analysis comprise a wide variety of health plan types and formularies and the study period encompassed the 
launch date of the first available DPP-4i. This ensured that the results were more generalizable than single payer databases or shorter time periods, which might be subject to systematic prescribing biases related to specific DPP-4i agents. The newuser cohort design, with the index date through the end of the follow-up period clearly anchored to medication exposure, is the default methodology recommended by the Agency for Healthcare Quality and Research for observational comparative effectiveness research. ${ }^{26}$ In terms of design, this study was limited to only those individuals with commercial health coverage or private Medicare supplemental coverage. Consequently, results of this analysis may not be generalizable to patients with other insurance or without health insurance coverage, and may differ from what would be observed in patients outside of the US. The MarketScan Research Databases rely on administrative claims data for clinical detail. These data are subjected to data coding limitations and data entry error. In addition, inaccuracy in diagnoses and measurement of treatment exposure in claims databases may introduce a dilution of associations.

The current analysis does not address the separate issue of whether serum DPP-4 levels may be a relevant biomarker for predicting response to asthma therapeutics targeting IL-13. IL-13 has been implicated as a central mediator of asthma pathophysiology, driving airway inflammation, remodeling, and hyper-responsiveness. In vitro experiments have shown that IL-13 induces DPP-4 expression in airway epithelial cells, suggesting that DPP-4 serum levels might identify asthma patients who have an enhanced benefit to anti-IL-13 therapy. A clinical trial has shown that increased serum DPP-4 levels predicted a beneficial response to tralokinumab, an anti-IL-13 monoclonal antibody, in terms of reducing exacerbations, improving lung function, and alleviating symptoms. ${ }^{27}$ It is also important to note that the DPP-4i agents under study did not include inhaled formulations, and the study did not directly measure the impact of local pulmonary exposure to DPP-4is. The pharmacokinetics of pulmonary exposure rather than oral systemic exposure may produce different results, and also warrants future study.

In summary, asthma patients newly initiating a DPP-4i for the treatment of T2DM do not differ with respect to the probability of achieving asthma control as compared to similar patients newly initiating non-DPP-4i antidiabetes medications.

\section{Acknowledgments}

The authors acknowledge Koustubh Ranade (MedImmune, Gaithersburg, MD, USA) and Stephen Johnston, a member of the steering committee who was employed by Truven Health Analytics at the time the study was conducted, for their contributions to this study. Truven Health Analytics, an IBM Company, received funding from AstraZeneca in relation to this study.

This work was previously presented as a poster at the annual international conference of the American Thoracic Society, May 19-24, 2017, Washington, DC (Colice G, et al. The Effect of Dipeptidyl-Peptidase-4 Inhibitors on Asthma Control: An Administrative Database Study to Evaluate a Potential Pathophysiological Relationship. Am J Respir Crit Care Med. 2017;195:A3050).

\section{Disclosure}

Gene Colice, Maria Gerhardsson de Verdier, Karma Rabon-Stith, and Christopher Ambrose are employees of AstraZeneca. Katherine Cappell, Debra E Irwin, Paul Juneau, and Anna Vlahiotis are employees of Truven Health Analytics, an IBM Company. David Price has board membership with Aerocrine, Amgen, AstraZeneca, Boehringer Ingelheim, Chiesi, Mylan, Mundipharma, Napp, Novartis, and Teva Pharmaceuticals; consultancy agreements with Almirall, Amgen, AstraZeneca, Boehringer Ingelheim, Chiesi, GlaxoSmithKline, Mylan, Mundipharma, Napp, Novartis, Pfizer, Teva Pharmaceuticals, and Theravance; grants and unrestricted funding for investigator-initiated studies (conducted through Observational and Pragmatic Research Institute Pte Ltd) from Aerocrine, AKL Research and Development Ltd, AstraZeneca, Boehringer Ingelheim, British Lung Foundation, Chiesi, Mylan, Mundipharma, Napp, Novartis, Pfizer, Respiratory Effectiveness Group, Teva Pharmaceuticals, Theravance, UK National Health Service, Zentiva; payment for lectures/speaking engagements from Almirall, AstraZeneca, Boehringer Ingelheim, Chiesi, Cipla, GlaxoSmithKline, Kyorin, Mylan, Merck, Mundipharma, Novartis, Pfizer, Skyepharma, and Teva Pharmaceuticals; payment for manuscript preparation from Mundipharma and Teva Pharmaceuticals; payment for the development of educational materials from Mundipharma and Novartis; payment for travel/accommodation/meeting expenses from Aerocrine, AstraZeneca, Boehringer Ingelheim, Mundipharma, Napp, Novartis, and Teva Pharmaceuticals; funding for patient enrolment or completion of research from Chiesi, Novartis, Teva Pharmaceuticals, and Zentiva; stock/stock options from AKL Research and Development Ltd which produces phytopharmaceuticals; owns $74 \%$ of the social enterprise Optimum Patient Care Ltd (Australia, Singapore, and UK) and 74\% of Observational and Pragmatic Research 
Institute Pte Ltd (Singapore); and is peer reviewer for grant committees of the Efficacy and Mechanism Evaluation programme, and Health Technology Assessment. All authors are members of the steering committee. The authors report no other conflicts of interest in this work.

\section{References}

1. Nieto-Fontarigo JJ, Gonzalez-Barcala FJ, San Jose E, Arias P, Nogueira M, Salgado FJ. CD26 and asthma: a comprehensive review. Clinic Rev Allerg Immunol. Epub 2016 Aug 25.

2. Wagner L, Klemann C, Stephan M, von Horsten S. Unravelling the immunological roles of dipeptidyl peptidase 4 (DPP4) activity and/or structural homologue (DASH) proteins. Clin Exp Immunol. 2016;184(3):265-283.

3. Zhong J, Gong Q, Gound A, Srinivasamaharaj S, Rajagopalan S. Recent advances in dipeptidyl-peptidase-4 inhibition therapy: Lessons from the bench and clinical trials. J Diabetes Res. 2015;2015:606031

4. Mamgain S, Mathur S, Kothiyal P. Immunomodulatory activity of DPP4. J Pharmacol Clin Toxicol. 2013;1:1006.

5. Omar B, Ahrén B. Pleiotropic mechanisms for the glucose-lowering action of DPP-4 inhibitors. Diabetes. 2014;63(7):2196-2202.

6. Struyf S, Proost P, Schols D, et al. CD26/dipeptidyl-peptidase IV downregulates the eosinophil chemotactic potency, but not the anti-HIV activity of human eotaxin, by affecting its interaction with CC chemokine receptor 3. J Immunol. 1999;162(8):4903-4909.

7. Forsmann U, Stoetzer C, Stephan M, et al. Inhibition of CD26/dipeptidyl peptidase IV enhances CCL11/eotaxin-mediated recruitment of eosinophils in vivo. J Immunol. 2008;181(2):1120-1127.

8. Proost P, De Meester I, Schols D, et al. Amino-terminal truncation of chemokines by CD26/dipeptidyl-peptidase IV. Conversion of RANTES into a potent inhibitor of monocyte chemotaxis and HIV-1-infection. J Biol Chem. 1998;273(13):7222-7227.

9. Yan S, Gebner R, Dietel, Schmiedek U, Fan H. Enhanced ovalbumininduced airway inflammation in CD26/- mice. Eur J Immunol. 2012; 42(2):533-540.

10. Nader MA. Inhibition of airway inflammation and remodelling by sitagliptin in murine chronic asthma. Inter Immunopharmacol. 2015;29(2):761-769.

11. Shiobura T, Chibana K, Watanabe T, et al. Dipeptidyl peptidase-4 is highly expressed in bronchial epithelial cells of untreated asthma and it increases cell proliferation along with fibronectin production in airway constitutive cells. Respir Res. 2016;17:28.

12. van der Velden VH, Wierenga-Wolf AF, Adriaansen-Soeting WC, Overbeek SE, Möller GM, Hoogsteden HC, Versnel MA. Expression of aminopeptidase $\mathrm{N}$ and dipeptidyl peptidase IV in the healthy and asthmatic bronchus. Clin Exp Allergy. 1998;28(1):110-120.
13. Lun SW, Wong CK, Ko FW, Hui DS, Lam CW. Increased expression of plasma and CD4+ T lymphocyte costimulatory molecule CD26 in adult patients with allergic asthma. J Clin Immunol. 2007;27(4): 430-437.

14. Giovannini-Chami L, Marcet B, Moreilhon C, et al. Distinct epithelial gene expression phenotypes in childhood respiratory allergy. Eur Respir J. 2012;39(5):1197-1205.

15. Roche N, Postma DS, Colice G, et al. Differential effects of inhaled corticosteroids in smokers/ex-smokers and nonsmokers with asthma. Am J Respir Crit Care Med. 2015;191(8):960-964.

16. Israel E, Roche N, Martin RJ, et al. Increased dose of inhaled corticosteroid versus add-on long-acting $\beta$-agonist for step-up therapy in asthma. Ann Am Thorac Soc. 2015:12(6):798-806.

17. Colice, G, Martin RJ, Israel E, et al. Asthma outcomes and costs of therapy with extrafine beclomethasone and fluticasone. J Allergy Clin Immunol. 2013;132(1):45-54.

18. Price D, Thomas M, Haughney J, et al. Real-life comparison of beclometasone dipropionate as an extrafine- or larger-particle formulation for asthma. Respir Med. 2013;107(7):987-1000.

19. Jacob C, Haas JS, Bechtel B, Kardos P, Braun S. Assessing asthma severity based on claims data: a systematic review. Eur J Health Econ. 2017;18(2):227-241.

20. Holman R. Metformin as first choice in oral diabetes treatment: the UKPDS experience. Journ Annu Diabetol Hotel Dieu. 2007:13-20.

21. Chang HY, Weiner JP, Richards TM, Bleich SN, Segal JB. Validating the adapted Diabetes Complications Severity Index in claims data. $\mathrm{Am}$ J Manag Care. 2012;18(11):721-726.

22. Deyo RA, Cherkin DC, Ciol MA. Adapting a clinical comorbidity index for use with ICD-9-CM administrative databases. $J$ Clin Epidemiol. 1992;45(6):613-619.

23. Adams RJ, Wilson DH, Taylor AW, et al. Coexistent chronic conditions and asthma quality of life: a population-based study. Chest. 2006;129(2):285-291.

24. Travers J, Marsh S, Williams M, et al. External validity of randomised controlled trials in asthma: to whom do the results of the trials apply? Thorax. 2007;62(3):219-223.

25. Price D, Wilson AM, Chisholm A, Rigazio A, Burden A, Thomas M, King C. Predicting frequent asthma exacerbations using blood eosinophil count and other patient data routinely available in clinical practice. J Asthma Allergy. 2016;9:1-12.

26. Velentgas P, Dreyer NA, Nourjah P, Smith SR, Torchia MM, editors. Developing a Protocol for Observational Comparative Effectiveness Research: A User's Guide. Rockville, MD: Agency for Healthcare Research and Quality; 2013.

27. Brightling CE, Chanez P, Leigh R, et al. Efficacy and safety of tralokinumab in patients with severe uncontrolled asthma: a randomised, double-blind, placebo-controlled, phase $2 \mathrm{~b}$ trial. Lancet Respir Med. 2015;3(9):692-701.
Pragmatic and Observational Research

\section{Publish your work in this journal}

Pragmatic and Observational Research is an international, peer-reviewed, open access journal that publishes data from studies designed to reflect more closely medical interventions in real-world clinical practice compared with classical randomized controlled trials (RCTs). The manuscript management system is completely online and includes a very quick and fair peer-review
Dovepress

system. Visit http://www.dovepress.com/testimonials.php to read real quotes from published authors. 